\title{
Afecções intestinais em bovinos
}

José Augusto Bastos Afonso

Clínica de Bovinos de Garanhuns, Universidade Federal Rural de Pernambuco (UFRPE), Garanhuns, PE, Brasil e-mail: jose.basilva@ufrpe.br

\section{Introdução}

A atual demanda por alimento causada pelo aumento da população humana tem forçado os sistemas de produção de ruminantes a modificar o teor de fibra na dieta por maiores proporções de grãos. Esta maior mudança na dieta de ruminantes tem sido feita de maneira mais intensa durante as últimas décadas, em sistemas de produção comercial, com a finalidade de maximizar a eficiência produtiva. A incorporação na alimentação de altas proporções de carboidratos de rápida degradabilidade não está apenas contribuindo para a ocorrência de doenças, mas causando outros efeitos colaterais em resposta ao declínio na proporção do conteúdo da fibra na dieta.

Modernos sistemas de produção que incluem algumas práticas e muitas modificações na alimentação durante diferentes estágios na produção, principalmente em vacas de leite, como o uso de rações com elevada densidade energética, modificam o padrão da dinâmica microbiana ruminal, elevando os riscos para a ocorrência de doenças metabólicas e digestivas. Outras condições a se considerar para que estes transtornos incidam são observadas em regiões como o semiárido do Nordeste, onde a escassez e irregularidade acentuada na distribuição de chuvas, tanto no tempo quanto no espaço, com a ocorrência de longos períodos de estiagem praticamente determinam a obrigatoriedade de suplementação de vacas leiteiras nos sistemas de produção nesta região, como a oferta de forragens de baixa qualidade, o uso de alimentos concentrados, o emprego de resíduos da agroindústria, na sua maioria de pouca digestibilidade, limitações na disponibilidade de água de qualidade, associados ao estrese térmico e a intensificação no manejo dos animais em produção.

A ocorrência de distúrbios digestivos em bovinos, principalmente naqueles com características de produção leiteira, chega a representar algo em torno de $18 \%$ de todos os casos atendidos na Clínica de Bovinos. Porém há de se relatar que a ocorrência das várias condições clínicas comprometendo a fisiologia intestinal em bovinos, de natureza funcional, intraluminal ou extraluminal, e estrangulação, é proporcionalmente muito inferior aos casos dos distúrbios relatados acometendo os pré-estômagos e abomaso, representando algo em 
torno de 15\% das doenças do sistema digestório. Desta forma, na nossa rotina clínica, as principais enfermidades acometendo o intestino delgado e grosso são várias, destacando-se com maior frequência obstruções por fitobezoários, dilatação do ceco, intussuscepção, vólvulo e torção intestinal. Serão abordadas as mais frequentes, com detalhes nos aspectos dos achados clínicos e terapêuticos.

\section{Obstrução intestinal por fitobezoários}

As obstruções causadas por fitobezoários podem ser comuns em áreas onde a alimentação é constituída por forragem rica em fibra de pouca digestibilidade (com elevado teor de lignina), sendo a principal causa de obstrução intraluminal em bovinos. $\mathrm{Na}$ região Nordeste do Brasil, este distúrbio é observado com maior frequência nos meses de verão ou quando ocorre um período de maior estiagem, onde a escassez de fibra de qualidade e o acesso à água são restritos aos animais. A obstrução causada por fitobezoários surge, quase sempre, em algum segmento do jejuno, mas também no duodeno, provocando diminuição na motilidade gastrintestinal. Esta condição provoca distensão dos pré-estômagos e abomaso, devido ao refluxo do conteúdo gastrintestinal.

A evolução da doença é normalmente de aguda à subaguda. Os sinais clínicos encontrados com maior frequência são apatia, desidratação, taquicardia e anorexia. A temperatura e a frequência respiratória se mantém na maioria dos casos dentro da faixa de normalidade. Alguns bovinos manifestam sinais de cólicas. A dinâmica ruminal fica comprometida em frequência e amplitude, assim como o peristaltismo intestinal. Na percussão e prova do balotamento, auxiliada pela auscultação no flanco direito, geralmente constata-se a presença de líquido no interior das alças intestinais. No exame retal, é verificado que na maioria dos animais as fezes são escassas, pastosas, fétidas e com uma coloração acinzentada; em alguns casos, somente há muco, e em determinadas situações, é possível a palpação dos fitobezoários nos segmentos do intestino delgado. Com relação aos exames laboratoriais, na análise do fluído ruminal há um comprometimento da fauna e flora microbiana ruminal e, dependendo da localização da obstrução, a concentração do teor de cloretos é elevada (> $30 \mathrm{mEq} / \mathrm{L}$ ). No hemograma, um quadro de leucocitose por neutrofilia, com desvio para esquerda regenerativo e hiperfibrinogenia, pode ser encontrado.

Quando diagnosticado este tipo de obstrução, a conduta terapêutica empregada é a celiotomia na fossa paralombar direita, com exploração do abdômen e exteriorização do segmento intestinal onde há a obstrução, e fluidoterapia (soro fisiológico, solução de Ringer/via intravenosa) para correção da desidratação e equilíbrio ácido-básico. No transoperatório é administrada oxitetraciclina solução (10 mg/kg PV) na cavidade abdominal e, posteriormente, oxitetraciclina de longa ação (10 mg/kg) a cada 72 horas/uma semana e AINES (Meloxican, flunexin neglumine), por via intravenosa, durante três dias, com intervalo de 24 horas. A terapia de suporte deve ser feita com aplicações de soluções de cálcio e uso de procinéticos (metoclopramida 0,1 a $0,5 \mathrm{~mL} / \mathrm{kg}$ / PV) por 24 a 48 horas para melhorar a motilidade. A administração de fluído ruminal (10 a 20 litros) durante as primeiras 48 horas após a realização da cirurgia auxilia a recomposição da microbiota. Em geral, o animal tende a eliminar fezes entre 24 e 48 horas após o procedimento. Nos casos de obstrução por fitobezoários, a recuperação cirúrgica tem sido bem favorável, principalmente quando diagnosticado precocemente e sem haver contaminação do abdômen.

\section{Intussuscepção/Vólvulo e torção}

Obstruções do intestino delgado, tais como intussuscepção e vólvulo, são pouco frequentes e de ocorrência mais rara em bovinos adultos, tendo maior frequência em bezerros com idade inferior a dois meses.

A intussuscepção é a invaginação de um segmento do intestino para dentro do lúmen do segmento adjacente. A obstrução intestinal ocorre devido à oclusão do lúmen intestinal pelo intenso edema que é verificado na área lesada. 0 suprimento sanguíneo fica comprometido, resultando em dor abdominal e isquemia no local, podendo ocorrer, consequentemente, peritonite. Embora a causa primária seja raramente identificada, a 
intussuscepção pode ocorrer secundária à enterite, parasitismo intestinal, mudanças abruptas na dieta, granuloma mural ou abscesso, neoplasias, hematoma mural e administração de drogas que afetam a motilidade intestinal. A maioria destes casos ocorre na porção distal do jejuno e proximal ao íleo, que é a região do intestino suspensa pela borda do mesentério, onde o mesmo é longo e mais móvel. Por isso, justifica-se sua maior ocorrência em bezerros (mais jovens), devido ao fato de o mesentério ser mais fino e, com isso, não proporcionar o mesmo grau de suporte como nos bovinos adultos.

0 vólvulo é a rotação de um segmento do intestino delgado sobre o seu eixo, criando uma obstrução e estrangulação do suprimento sanguíneo. Todavia o local mais frequentemente observado é o segmento distal do jejuno e o íleo. A lesão resulta numa distensão intestinal - devido ao acúmulo de líquido e conteúdo intestinal -, acarretando um comprometimento vascular, necrose intestinal e, eventualmente, morte. Esta condição ocorre de forma muito esporádica em bovinos, sendo mais frequente em gado de leite do que em gado de corte, e com maior ocorrência em animais jovens, porém sua causa não é bem definida. Enquanto a torção se faz sobre a alça mesentérica, envolvendo partes do intestino delgado e grosso, é de ocorrência rara.

A evolução na maioria dos casos é aguda e os sinais clínicos iniciais evidentes são de cólicas (escoiceia o abdômen, deita e levanta-se constantemente, grunhindo), de curta duração (8 a 12 horas) e de maior intensidade em animais com torção. Após este período, estas são menos intensas, e constata-se ainda apatia, desidratação, anorexia, hipomotilidade gastrintestinal, distensão abdominal, estando o intestino distendido por fluídos e identificado pela sucussão e auscultação do quadrante inferior direito do abdômen. $\mathrm{Na}$ maioria dos casos a frequência cardíaca está elevada em razão da gravidade da lesão circulatória. 0 exame retal é de grande auxílio para o diagnóstico na maioria dos casos de obstrução, através do qual, na intussuscepção e no vólvulo, os segmentos do intestino delgado comprometidos podem ser sentidos, juntamente com algumas alças distendidas e tensão do mesentério no lado direito do abdômen. Alguns animais sentem dor quando esta conduta é realizada. A produção de fezes tende a diminuir até se tornarem ausentes (sinal de braço positivo); porém em alguns casos de intussuscepção e de vólvulo, há a presença de um muco sanguinolento, não como melena, bastante untuoso e de cor vermelha escura. Estes achados, quase patognomônicos, são considerados como um sinal no diagnóstico destes dois tipos de afecções intestinais. É claro que as variações na intensidade dos sinais obviamente dependem da evolução, do tipo da obstrução e da gravidade da lesão. Na análise do fluído ruminal, verifica-se comprometimento da dinâmica da microbiota e a elevação no teor de cloreto (> $30 \mathrm{mEq} / \mathrm{L}$ ), enquanto o hemograma, na maioria dos casos, revela leucocitose com desvio à esquerda. 0 diagnóstico da distinção destas entidades é por laparotomia exploratória

A condição clínica do paciente e a natureza da obstrução permitem discutir o tipo de conduta a ser tomada. Na maioria das vezes, principalmente em se tratando de vólvulo/torção e da condição zootécnica, o abate pode ser a opção mais econômica para animais de valor comercial. Entretanto, se o diagnóstico da obstrução requer o tratamento cirúrgico - a celiotomia da fossa sublombar direita este pode ser realizado, de forma imediata, no início do curso da enfermidade. 0 procedimento na maioria das vezes é realizado em estação, porém, se o animal tende a deitar-se, a cirurgia pode ser realizada com o animal em decúbito lateral esquerdo. A fluidoterapia (IV) deve ser empregada (soro fisiológico, solução de Ringer) para restabelecer o volume normal circulante, corrigindo a desidratação e o equilíbrio ácido-básico e evitando o risco de choque. 0 uso de analgésicos nas primeiras 48 horas é prudente. 0 emprego de antibióticos de largo espectro se faz necessário por sete a 10 dias. 0 uso de drogas procinéticas deverá ser empregado. 0 paciente deverá receber fluído ruminal, alimento (forragem e um pouco de ração de boa qualidade) e água à vontade, cuja finalidade é estabelecer a microflora normal do rúmen e a motilidade gastrintestinal. Não apresentando complicações no pós-operatório, o paciente deve defecar entre 24 e 48 horas após a cirurgia. Retornando o apetite, a dinâmica digestiva e mantendo-se esta evolução clínica nos primeiros cinco a sete dias, o prognóstico é favorável.

0 prognóstico varia muito em função da natureza da obstrução, duração e severidade da 
lesão vascular. Os índices de recuperação , em casos de correção cirúrgica, na intussuscepção e vólvulo foram inferiores a $50 \%$.

\section{Dilatação do ceco}

A dilatação do ceco (DC), embora seja uma desordem digestiva pouco frequente em relação aos outros tipos de afecções do trato gastrointestinal em bovinos, constitui uma causa comum de disfunção digestiva, principalmente em bovinos de leite.

A teoria proposta mais sugestiva para explicar este distúrbio fermentativo é a que a atonia do órgão permite o acúmulo de gás e que, consequentemente, há dilatação e, em certas situações, vólvulo. Normalmente o ceco é responsável pela digestão de 10 a $17 \%$ da celulose e $20 \%$ dos carboidratos solúveis consumidos pelos bovinos. Os ácidos graxos voláteis (AGV) produzidos no ceco são absorvidos através do epitélio cecal e aumentam a absorção com o declínio do $\mathrm{pH}$. A produção de AGV eleva-se e o pH e a motilidade diminuem no ceco quando a dieta fornecida é modificada de uma constituída de forragem para uma dieta rica em concentrado. Portanto a elevação na concentração destes AGV pode acarretar uma atonia do ceco, e principalmente o butirato é considerado o inibidor mais potente. Estas alterações acarretam dilatação cecal e criam a possibilidade para a torção.

Fatores adicionais, como a hipocalcemia, a endotoxemia secundária a metrite ou mastite, podem predispor à DC. Este tipo de desordem tende a ocorrer com maior frequência em bovinos adultos, no início da lactação, embora tenha sido relatada em outras fases da lactação. A DC também foi observada em bezerros e bois. A maior ocorrência dos casos é verificada em animais mantidos em regime intensivo.

Clinicamente, caracteriza-se por inapetência, queda na produção leiteira e diminuição na quantidade de fezes. Na percussão e auscultação, há um ping (tilintar) sobre a porção superior do flanco direito e projeção com distensão reconhecível da víscera na palpação retal.

Na DC, o clínico deverá decidir se o tratamento médico é o mais adequado ou se haverá a necessidade de uma exploração cirúrgica. Em nossa experiência na Clínica de Bovinos, na maioria dos casos a terapia cirúrgica é a mais empregada. 0 tratamento cirúrgico consiste em uma laparotomia na região do flanco direito, que permite a exposição do ceco, e a correção usualmente envolve uma tiflotomia, com retirada do excesso do conteúdo alimentar. Em algumas situações há também, simultaneamente, compactação do cólon, que dificulta a recuperação, provocando reincidência da dilatação do ceco em alguns casos. Em casos graves em que há necrose da parede do ceco, uma parcial tiflectomia pode ser necessária.

Uma vez a DC corrigida, no pós-operatório uma terapia de suporte é indicada por alguns dias para corrigir a hidratação e o déficit eletrolítico, fazendo-se uso de fluidoterapia oral ou intravenosa, antinflamatórios não esteroides e laxativos (suco de mandacaru). Soluções de cálcio são importantes e também empregadas. 0 uso de procinéticos pode ser aplicado por 48 horas, para melhorar a motilidade. 0 tratamento de algumas doenças concomitantes, como a cetose, metrite e mastite, deve ser realizado. Caso o conteúdo ruminal esteja comprometido, a reposição de fluído ruminal é uma boa terapia auxiliar. A palpação retal deverá ser realizada 24 horas após a cirurgia para avaliar o grau de distensão (regresso) do órgão. A antibioticoterapia é indicada por sete dias. Em casos não complicados, os bovinos retomam o apetite e voltam a defecar grande quantidade de fezes amolecidas dentro de 24 horas pós-cirurgia. 0 prognóstico é bom para os pacientes com DC, com índices de recuperação clínica podendo chegar a $80 \%$ dos casos. As principais complicações que podem surgir incluem a reincidência em aproximadamente $10 \%$ dos casos e a possibilidade de peritonite naqueles casos com procedimentos cirúrgicos complicados.

De uma maneira geral, em relação às enfermidades comentadas e suas respectivas condutas, os animais acometidos e tratados cirurgicamente deverão ser mantidos em baias ou piquetes limpos, com água e comida adequada e fresca. É necessário que a ferida cirúrgica esteja limpa e seja sempre verificada. É importante que a observação quanto à evolução do quadro clínico do animal em termos de recuperação seja diária e por no mínimo uma semana. Os exames laboratoriais (hemograma, provas de função ruminal, etc.) auxiliam bastante neste período. A terapia antidor no pós-operatório deve ser praticada, pois auxilia na recuperação do paciente. Bovinos não são 
mais resistentes do que cavalos a infecções cirúrgicas, e se os riscos de contaminação existiram, todos os esforços deverão ser realizados para prevení-las.

É necessário relatar que enfermidades de menor ocorrência, como as úlceras de duodeno e jejuno, compactação de colón e colite ulcerativa, síndrome jejuno hemorrágica, além das causas extraluminais, como abscesso de mesentério, linfosarcomas e granulomas por tuberculose, têm a sua importância, pois apesar de serem registros pontuais, estão relacionadas com práticas inadequadas no manejo alimentar e sanitário que estão comprometendo a sanidade do rebanho.

\section{Referências}

Afonso JAB, Mota IO, Costa NA, Mendonça CL, Souza MI. Terapia e evolução clínica em bovinos com dilatação do ceco: análise de 14 casos. Rev Bras Med Vet. 2006;28(4):172-6.

Afonso JAB, Costa NA. Doenças não transmissíveis do trato digestivo dos ruminantes: obstrução intestinal em bovinos, p.370-371. In: Riet-Correa F, Schild AL, Lemos RAA, Borges JRJ (Eds). Doenças de Ruminantes e EqUídeos. Vol 2. 3aㅡ ed. Santa Maria: Palloti;2007.

Afonso JAB, Pereira ALL, Vieira ACS, Mendonça CL, Costa NA, Souza MI. Alterações clínicas e laboratoriais na obstrução gastrintestinal por fitobezoários em bovinos. Rev Bras Saúde Prod An. 2008;9(1):91-102.

Afonso JAB, Souza JCA, Guaraná ELS, Mendonça CL, Costa NA, Rego RO. Compactação do cólon em vaca. Relato de caso. Cienc Anim Bras. 2009;(Supl. 1). Disponível em: https://tinyurl.com/yb2zlpxz.

Braun U, Beckmann C, Gerspach C, Hässig M, Muggli E, Knubben-Schweizer G, et al. Clinical findings and treatment in cattle with caecal dilatation. BMC Vet Res. 2012;8:75.

Braun U, Schmid T, Muggli E, Steininger K, Previtali M, Gerspach C, et al. Clinical findings and treatment in 63 cows with haemorrhagic bowel syndrome. Schweiz Arch Tierheilkd. 2010;152(11):515-22.
Cajueiro JFP, Gomes RC, Fereira LEPA, Costa NA, Coutinho LT, Afonso JAB. Abscesso mesentérico como causa de obstrução intestinal em um bovino leiteiro. Vet Zootec. 2011;18(4 Supl. 3):520-2.

Clínica de Bovinos de Garanhuns. Livro de registro dos casos clínicos em ruminantes. Garanhuns: Universidade Federal Rural de Pernambuco; 2017.

Constable PD, St Jean G, Hull BL, Rings DM, Morin DE, Nelson DR. Intussusception in cattle: 336 cases (19641993). J Am Vet Med Assoc. 1997;210(4):531-6.

Desrochers A, Anderson DE. Intestinal Surgery. Vet Clin North Am Food Anim Pract. 2016;32(3):645-71.

Ferreira MA, Silva FM, Bispo SV, Azevedo M. Estratégias na suplementação de vacas leiteiras no semi-árido do Brasil. R Bras Zootec. 2009;38(spe.):322-9.

Francoz D, Guard CL. Obstructive intestinal diseases. In: Smith BP. Large animal internal medicine. $5^{\circ}$ ed. St. Louis: Mosby; 2015. p. 820-4.

Fubini SL, Erb HN, Rebhun WC, Horne D. Cecal dilatation and volvulus in dairy cows: 84 cases (1977-1983). J Am Vet Med Assoc. 1986;189(1):96-9.

Fubini SL, Ducharme NG. Farm animal surgery. St. Louis: Saunders; $2004.607 \mathrm{p}$.

Marques LC, Cattelan JW, Macoris DG, Portugal ES, Cadioli FA. Estudo clínico, cirúrgico e anatomopatológico de intussuscepção em quatro bovinos. Arq Bras Med Vet Zootec. 2001; 53(1):52-7.

Nagaraja TG, Galyean ML, Cole NA. Nutrition and disease. Vet Clin North Am Food Anim Pract. 1998;14(2):257-77.

Nascimento EM, Medeiros RMT, Simões SVD, RietCorrea F. Compactação ruminal e obstrução intestinal em bovinos, associadas ao consumo de Agave sisalana Perrine (Agavaceae). Pesq Vet Bras. 2016;36(8):719-23.

Ortolani EL, July JR, Feitosa LF. A rare case of intussusception in a dairy cow. Braz J Vet Res Anim Sci. 1995;32(1):27-30. 
Pires PS, Uribe JAZ, Carvalho AU, Silva ROS, Salvarani FM, Meneses RM, et al. Jejunal hemorrhage syndrome in a Zebu cow in Brazil. Cienc Rural. 2015;45(8):1476-9.

Radostits O, Gay C, Hinchcliff $\mathrm{K}$, Constable P. Veterinary Medicine: A textbook of the diseases of cattle, horses, sheep, pigs and goats. 10a ed. Edimburgo: Saunders; 2007.

Silanikove N. Effects of water scarcity and hot environment on appetite and digestion in ruminants: a review. Livest Prod Sci. 1992;30(3):175-94.

Silva Filho AP, Afonso JAB, Souza JCA, Costa NA, Mendonça CL. Clinical and pathological analysis in 20 cases of intussusception in cattle. Vet Zootec. 2010;17:422-31.

Silva RJ, Afonso JAB, Costa NA, Mendonça CL. Dilatação do ceco em bezerros: relato de casos. Rev Bras Cienc Vet. 2014;21(2):76-81.

Tarso SGS, Oliveira D, Afonso JAB. Ruminants as Part of the Global Food System: How Evolutionary Adaptation Sand Diversity of the Digestive System Brought them to the Future. J Dairy Vet Anim Res. 2016;3(5):00094. 